\title{
Assessing the quality of reports of randomized trials in pediatric complementary and alternative medicine David Moher*1,2, Margaret Sampson ${ }^{1}$, Kaitryn Campbell ${ }^{3}$, William Beckner ${ }^{4}$, Leah Lepage ${ }^{1}$, Isabelle Gaboury ${ }^{1}$ and Brian Berman ${ }^{4}$
}

Address: ${ }^{1}$ Thomas C. Chalmers Centre for Systematic Reviews, Children's Hospital of Eastern Ontario Research Institute, Canada, ${ }^{2}$ Department of Pediatircs, University of Ottawa, Canada, ${ }^{3}$ Faculty of Information and Media Studies, University of Western Ontario, Canada and ${ }^{4}$ University of Maryland, Complementary Medicine Program, USA

E-mail: David Moher* -dmoher@uottawa.ca; Margaret Sampson - msampson@uottawa.ca; Kaitryn Campbell - kaitryn_Campbell@hotmail.com; William Beckner - Mac@compmed.umm.edu; Leah Lepage - llepage@uottawa.ca; Isabelle Gaboury - igaboury@cheo.on.ca; Brian Berman - BBerman@compmed.umm.edu

${ }^{*}$ Corresponding author

Published: 27 February 2002

BMC Pediatrics 2002, 2
Received: 10 December 200I

Accepted: 27 February 2002

This article is available from: http://www.biomedcentral.com//47I-243I/2/2

(C) 2002 Moher et al; licensee BioMed Central Ltd. Verbatim copying and redistribution of this article are permitted in any medium for any purpose, provided this notice is preserved along with the article's original URL.

\begin{abstract}
Objective: To evaluate the quality of reports of complementary and alternative medicine (CAM) randomized controlled trials (RCTs) in the pediatric population. We also examined whether there was a change in the quality of reporting over time.

Methods: We used a systematic sample of $25 \mathrm{I}$ reports of RCTs that used a CAM intervention. The quality of each report was assessed using the number of CONSORT checklist items included, the frequency of unclear allocation concealment, and a 5-point quality assessment instrument.

Results: Nearly half (40\%) of the CONSORT checklist items were included in the reports, with an increase in the number of items included. The majority (81.3\%) of RCTs reported unclear allocation concealment with no significant change over time. The quality of reports achieved approximately $40 \%$ of their maximum possible total score as assessed with the Jadad scale with no change over time. Information regarding adverse events was reported in less than one quarter of the RCTs (22\%) and information regarding costs was mentioned in only a minority of reports (4\%).

Conclusions: RCTs are an important tool for evidence based health care decisions. If these studies are to be relevant in the evaluation of CAM interventions it is important that they are conducted and reported with the highest possible standards. There is a need to redouble efforts to ensure that children and their families are participating in RCTs that are conducted and reported with minimal bias. Such studies will increase their usefulness to a board spectrum of interested stakeholders.
\end{abstract}

\section{Introduction}

The use of complementary and alternative medicine (CAM) has become increasingly popular for both adult and pediatric populations. For example, in 1992, 11\% of a Montreal-based pediatric sample used CAM [1]. By 1997 , however, this figure had grown to $17 \%$ [2]. The 1994 National Population Health Survey suggested that $15 \%$ of Canadians (any age) had used CAM during the 
course of the preceding year [3]. By 1999, this figure had grown to roughly $70 \%$ [4]. Similar data has been reported for other jurisdictions $[5,6]$.

What is less clear is the quality of evidence for the use of these products and practices. Focusing our attention on reports of randomized controlled trials (RCTs) allows us to examine the gold standard for evaluating an intervention's effectiveness. This enables readers to judge the extent to which the results are internally valid and free of bias.

Within conventional medicine there is substantial evidence about the quality of reports of RCTs and the consequences of lower quality reporting. Schulz and colleagues have documented that only about a third of reports of RCTs adequately report allocation concealment [7]. Reports of inadequate allocation concealment, compared to those in which this information is adequately reported (i.e., adequate allocation concealment), exaggerate the estimates of an intervention's effectiveness by about $30 \%$, on average [8-10]. These investigations and others have typically focused on interventions such as pharmaceuticals and adult populations.

We are unaware of any systematic effort to examine the extent of bias in reports of CAM RCTs specifically targeted at pediatric populations. This is important because if CAM studies are not subjected to rigorous evaluations they may jeopardize the health of children and their families [11]. Our primary focus was to evaluate the quality of reports of RCTs in the pediatric population (PedCAM). As a secondary question we also examined whether there was a change in the quality of reporting over time.

\section{Methods}

We have previously described the assembly of a comprehensive database of PedCAM RCTs [12]. Briefly, after defining CAM we searched 13 bibliographic databases using one of three search strategies. We also identified RCTs from cited references of 47 PedCAM systematic reviews [13]. The search results were downloaded to a reference database and screened. After identifying the PedCAM RCTs, clusters of trials relating to specific disease conditions and intervention types were identified. Three members of the research team (DM, MS, LL) nominated clusters for further consideration. Our goal was to identify 300 trials for closer examination, ensuring broad coverage of diseases and interventions from the full set of identified reports.

Once these reports were retrieved we extracted descriptive information using a 17-item structured data collection form. The questions pertained to the type of CAM used, the condition under investigation (according to the Inter- national Classification of Disease - ICD-9), the number and gender of the included children, the number and type of outcomes used, information about the reporting of adverse events and whether the authors reported on any cost information. The complete questionnaire can be obtained from the authors.

We also completed a comprehensive quality assessment of each report using three methods. First, the revised CONSORT statement checklist [14] was modified so that multiple items were listed separately, which resulted in 32 items. Each item was assigned a yes or no response depending on whether or not the author had reported it. Second, the reporting of allocation concealment was assessed as adequate, inadequate, or unclear [8] Third, the Jadad scale [15], which contains two questions each on randomization and masking, and one question on reporting of dropouts and withdrawals, was used to assess quality. Each question contains a yes or no response option. In total, five points can be awarded with higher points indicating superior quality. Three reviewers (DM, LL and MS) completed all of these evaluations.

We did not conduct any formal training prior to evaluating the RCTs using any of the three methods. We have extensive experience using these methods and have previously conducted training with results indicating substantial agreement between raters [16]. Discrepancies were resolved by consensus between the three raters.

We compared the number of checklist criteria included in each report and the mean number of criteria included within each subheading as specified in the CONSORT checklist. We also assessed the percentage of studies that reported unclear allocation concealment and the specific item and overall quality score derived from the Jadad instrument. The number of CONSORT checklist items reported was compared over time (1970s, 1980s, 1990s, 2000s) using an analysis of variance. A similar approach was used to assess the individual components and overall total score of the Jadad scale. The percentage of unclear allocation concealment was evaluated using $\chi^{2}$ tests.

\section{Results}

Database searching identified 3580 citations. Of these 2975 were screened from which 1468 PedCAM RCTs were identified (Figure 1). We systematically sampled 301 of these reports for further study. Twenty reports failed to meet our eligibility criteria and a further 30 reports were not evaluated (Figure 1) leaving 251 reports from which descriptive information and quality assessment was completed (Table 1).

The reported objective of CAM intervention was to manage or minimize current symptoms in two thirds of the re- 


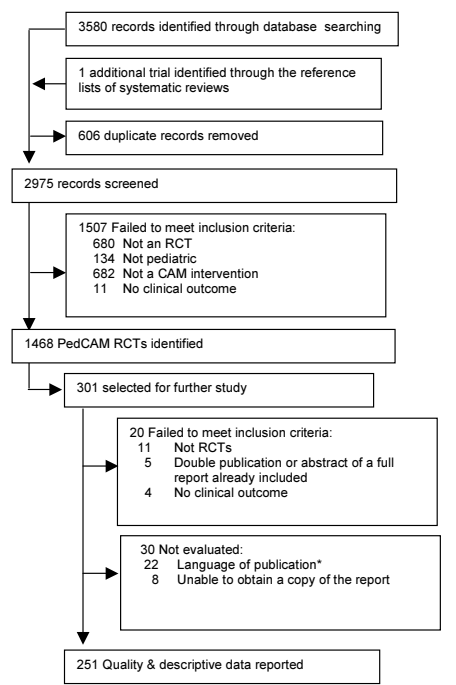

*Due to the language in which the paper was written, we were not able to read these papers in enough detail to reliably extract information: German (2), Hungarian (1), French (1), Japanese (2), Chinese (16). Note that there were some German and some French papers that we were able to include.

\section{Figure I}

Flow of citations and articles through the phases of screening and eligibility evaluation

ports $(66.7 \%)$ and more than a quarter of the RCTs were undertaken to help prevent disease $(32.5 \%)$. There were 157 reports involving fewer than 100 children (mean = 47.31; standard deviation $=24.14) ; 63$ reports included between 100 and 1000 children (mean = 298.38; standard deviation $=232.51$ ); and 9 reports included more than 1000 children (mean $=6766$; standard deviation $=$ 8402.72).

Forty percent (12.7 out of 32) of the CONSORT checklist items were included in the reports of PedCAM RCTs (Table 2$)$. There was an increase over time $(\mathrm{p}<0.001)$ in the number of checklist items included in the reports (Table 2 ). Between the 1980s, when 10.8 checklist items were reported, and the 1990s, when 13.4 checklist items were reported, there was a $24 \%$ increase in the number of checklist items reported $(\mathrm{p}=0.001$; mean difference $=$ 2.55 (95\% confidence interval: $-4.28,-0.81)$. There was a minor decrease in the 2000s in the number of CONSORT items reported.

The majority (83.1\%) of RCTs reported unclear allocation concealment (Table 3). We were unable to detect a change over time ( $\mathrm{p}=0.496$; Table 3 ). The quality of reports achieved approximately $40 \%$ of their maximum possible total score as assessed by using the Jadad scale (Table 3 ).
There was no improvement in the quality of reporting over time $(\mathrm{p}=0.174)$.

Information regarding adverse events was reported in less than one quarter of the RCTs (22.4\%). Similarly, information regarding costs (e.g., cost effectiveness) was mentioned in only a minority of reports $(4.5 \%)$.

\section{Discussion}

About one third of the CONSORT checklist items were included in reports of PedCAM RCTs. Although there is still considerable room for improvement in how these studies are reported, there has been a notable increase in the quality of their reports since the 1980s. The 24\% increase in the number of reported CONSORT items is encouraging and probably reflects important reductions in bias in the results of RCTs. Similar results were observed when the assessments focused on the adequacy of allocation concealment or the Jadad assessment. Unfortunately, these results also suggest that the validity of some of the PedCAM RCT results is probably questionable. This is particularly true if our attention focuses on aspects of how randomization was reported. Only about half of the reports documented how the random numbers were generated and three quarters of the reports had unclear allocation concealment.

Conducting a randomized trial is a complex series of tasks and it may not always be possible to minimize some potential biases. For example, double blinding (masking) is questionable both ethically and scientifically when performing surgical trials. However, in every single randomized trial it is always possible to ensure that the random numbers are appropriately generated (e.g., computer generation) and concealed from all parties involved in the trial until the child has been randomized (e.g., centralized randomization). High quality reports always include this information.

Our results indicate that the quality of reports of PedCAM RCTs may be lower than that found for conventional medicine. It is difficult to be more certain because the degree of journal overlap between different studies is unknown. In a recent report on the assessment of 77 RCTs published in 1998, in three high impact factor journals, the average number of CONSORT items included was 27.1 (out of 40) [16]. The average Jadad score was $62 \%$ of the maximum possible score and $39 \%$ of the reports had unclear allocation concealment. Linde and colleagues recently reported on the quality of 207 trials in homeopathy, herbs and acupuncture [17]. The average Jadad score ranged from about $40 \%$ (acupuncture) to $60 \%$ (herbs) of the maximum possible total score. The Jadad scores are similar to those observed in this study. However, the CONSORT results reported here are considerably lower than observed elsewhere. 
Table I: Conditions and Interventions selected for further study

\begin{tabular}{lcc}
\hline Condition & Original N & N after Exclusions \\
& & \\
Asthma & & 31 \\
Colic & 34 & 16 \\
Dermatological conditions (including scars) & 19 & 30 \\
Diarrhea & 36 & 49 \\
Malaria & $75^{*}$ & 20 \\
Migraine / headache & 21 & 18 \\
Interventions / Treatment modalities Biofeedback & 19 & 22 \\
Chinese Herbal Medicine & 27 & 6 \\
Chiropractic & $20 \%$ & 5 \\
Homeopathy & 7 & 6 \\
Massage or holding & 9 & 25 \\
TENS & 26 & 3 \\
Zinc & 8 & 39
\end{tabular}

* We examined only 49 of 75 diarrhea studies to avoid giving undue weight to this condition. \%Many of these were excluded based on language Note: some trials involved both a condition and a treatment chosen (i.e., chiropractic for colic) and so numbers to not total to $25 \mathrm{I}$.

Table 2: CONSORT checklist criteria included in $25 \mathrm{I}$ reports of complementary and alternative medicine randomized controlled trials in children published over four decades

\begin{tabular}{|c|c|c|c|c|c|}
\hline Total number of items & & & Decade & & \\
\hline & $1970 s(n=5)$ & $1980 s(n=47)$ & $1990 s(n=186)$ & $2000 s(n=13)$ & Total $(n=25 \mathrm{I})$ \\
\hline Title $=1, n \%$ & $2(40.0)$ & $29(61.7)$ & I 44 (77.4) & $12(92.3)$ & $187(74.5)$ \\
\hline Introduction $=1, n \%$ & $5(100.0)$ & $42(89.4)$ & $170(9 \mid .4)$ & II (84.6) & $228(90.8)$ \\
\hline Methods $=18$, mean $(s d)$ & $5.0(3.7)$ & $5.8(1.8)$ & $7.1(2.6)$ & $6.5(2.9)$ & $6.8(2.5)$ \\
\hline Results $=9$, mean $(s d)$ & $1.0(1.0)$ & $1.6(0.9)$ & $2.5(1.8)$ & $2.2(1.9)$ & $2.3(1.7)$ \\
\hline Discussion $=3$, mean $(s d)$ & $1.2(1.3)$ & $2.0(1.1)$ & $2.1(1.0)$ & $1.9(1.1)$ & $2.0(1.0)$ \\
\hline Total $=32$, mean $(\mathrm{sd})$ & $8.6(5.4)$ & $10.8(3.0)$ & $13.4(4.3)$ & $12.3(5.3)$ & $12.7(4.3)$ \\
\hline
\end{tabular}

Table 3: Quality of reports of 25 I complementary and alternative medicine randomized controlled trials in children using the Jadad assessment scale and the adequacy of allocation concealment

\begin{tabular}{|c|c|c|c|c|c|}
\hline \multirow[t]{2}{*}{ Total number of items } & \multicolumn{5}{|c|}{ Decade } \\
\hline & $1970 s(n=5)$ & $1980 s(n=47)$ & $1990 s(n=186)$ & $2000 s(n=13)$ & Total $(n=25 \mathrm{I})$ \\
\hline Randomization, mean (sd) & $0.2(0.8)$ & $0.7(0.7)$ & $1.0(0.6)$ & $1.2(0.6)$ & $0.9(0.6)$ \\
\hline Double-blinding, mean (sd) & $0.8(1.1)$ & $0.6(0.9)$ & $0.6(0.9)$ & $0.6(0.9)$ & $0.6(0.9)$ \\
\hline Withdrawals/dropouts, n (\%) & $2(40.0)$ & $12(25.5)$ & $89(47.8)$ & $2(15.4)$ & $105(4 \mid .8)$ \\
\hline Overall $=5$, mean $(s d)$ & $\mathrm{I} .4(\mathrm{I} .7)$ & $1.6(1.3)$ & $2.0(1.2)$ & $2.0(1.5)$ & $1.9(1.3)$ \\
\hline Unclear allocation concealment, $\mathrm{n}(\%)$ & $4(80.0)$ & $41(87.2)$ & $150(80.6)$ & $9(69.2)$ & $204(8 I .3)$ \\
\hline
\end{tabular}


One way to improve the quality of reporting of PedCAM RCTs is for more pediatric journals to endorse the CONSORT statement. There is evidence to suggest that journals using the CONSORT statement, compared to those not doing so, have higher quality reports of RCTs [15]. Of course examining the quality of reporting is 'after the fact' when the trial is already completed. CONSORT can also be used by granting agencies [18]; (Allan Bernstein, President of the Canadian Institutes of Health Research, personal communication) to encourage prospective investigators to improve the conduct of their RCTs.

It is not immediately clear why we observed lower quality scores in these reports. It is possible that the PedCAM community conducts fewer RCTs and is therefore less experienced. We have observed that prior to 1975 there were very few published reports of PedCAM RCTs [12], although there has been a sharp increase in the number of reports during the 1990s. These results might also reflect that the PedCAM community has been slower to train researchers in the appropriate conduct of RCTs.

Beyond examining the quality of these reports we were disappointed to find that so few reports mentioned anything about adverse events. This result is similar to that recently reported [19]. Although information on adverse events is extremely important authors have typically devoted less space to this information than to their names and affiliations [20]. Only about one in twenty reports mentioned anything about costs, such as a cost benefit analysis, in their reports. If clinicians and policy makers are to make decisions about the utility of CAM interventions for the pediatric population they will need more information than simply the efficacy of the intervention.

This study had a number of limitations. Our focus was on the quality of reporting of PedCAM RCTs. It is possible that the trials were appropriately conducted but had deficiencies in their reporting. Despite the paucity of data addressing this important question, the evidence that is available points in the direction of a reasonably good correlation between how investigators conduct their trials and how they are subsequently reported $[21,22]$. We did not take a random sample of all 1468 trials identified. It is possible that our sample does not reflect the total population and that these results cannot be generalized to all PedCAM RCTs. We selected the reports to broadly reflect the ICD categories and CAM interventions of the 1468 reports. We believe that our sampling approach although systematic is representative and enables us to generalize the observed results. The 2000s results need to be interpreted with caution and are probably not representative of the population, as there were only 13 reports. The small number probably reflects the delay in indexing the studies on electronic databases. We excluded 22 reports because they were written in languages other than English. It is possible that the quality of these reports differs in some systematic way from English language reports. Previous research suggests that the quality of RCTs reported in nonEnglish languages is similar to those reported in English [23].

Randomized trials are an important tool for evidence based health care decisions. If these studies are to be relevant in the evaluation of CAM interventions it is important that they are conducted and reported with the highest possible standards. There is a need to redouble efforts to ensure that children and their families are participating in RCTs that are conducted and reported with minimal bias. Such studies will increase their usefulness to a board spectrum of interested stakeholders.

\section{Competing interests}

None declared.

\section{Acknowledgements}

We thank Tammy Clifford for her expertise and guidance in the field of Complementary and Alternative Medicine, and Rose Marie Mongeon and Patricia Jackson for document retrieval.

\section{References}

I. Spigelblatt L, Laine-Ammara G, Pless IB, Guyver A: The use of alternative medicine by children. Pediatrics 1994, 94:8II-4

2. Ramsay S: In whose best interest is government-run health care? Fraser Forum. Fraser Institute. I I- / 3-200 I. Ref Type: Electronic Citation 1997

3. Millar W]: Use of Alternative Health Care Practitioners by Canadians. Canadian Journal of Public Health 1997, 88: I54-8

4. Berger E: Berger Population Health Monitor 21. 2000. Toronto, Hay Associates. Ref Type: Serial (Book, Monograph)

5. Eisenberg DM, Kessler RC, Foster C, Norlock FE, Calkins DR, Delbanco TL: Unconventional medicine in the United States: prevalence, costs and pattern use. $N$ Engl J Med I993, 328:24652

6. Eisenberg DM, Davis RB, Ettner SL, Appel S, Wilkey S, van Rompay $M$, et al: Trends in alternative medicine use in the United States, 1990-1997: results of a follow-up national survey. JAMA 1998, 280:1569-75

7. Schulz KF, Chalmers I, Grimes DA, Altman DG: Assessing the quality of randomization from reports of controlled trials published in obstetrics and gynecology journals. JAMA 1994, 272: $125-8$

8. Schulz KF, Chalmers I, Hayes RJ, Altman DG: Empirical evidence of bias: Dimensions of methodological quality associated with estimates of treatment effects in controlled trials. JAMA 1995, 273:408-12

9. Moher D, Pham B, Jones A, Cook DJ, Jadad AR, Moher M, et al: Does the quality of reports of randomised trials affect estimates of intervention efficacy reported in meta-analyses? Lancet 1998, 352:609-13

10. Kjaergård LL, Villumsen J, Gluud C: Reported methodological quality and discrepancies between large and smaller randomized trials. $B M J 200 I$

II. Ernst $E$ : The role of complementary and alternative medicine. BMJ 2000, 32I: II 33-5

12. Campbell K, Sampson M, Ajiferuke I, Moher D: Publication characteristics of randomized controlled trials in pediatric complementary and alternative medicine. Submitted for publication.

13. Moher D, Soeken K, Sampson M, Campbell K, Ben Perot L, Berman $B$ : Assessing the quality of reports of systematic reviews in pediatric complementary and alternative medicine. BMC Pediatrics 2002, 2:3 
14. Moher D, Schulz KF, Altman DG, for the CONSORT group: The CONSORT statement: revised recommendations for improving the quality of reports of parallel group randomized trials. Annals of Internal Medicine 200I, I34:657-662

15. Jadad AR, Moore RA, Carroll D, Jenkinson C, Reynolds DJ, Gavaghan $D$, et al: Assessing the quality of reports of randomized clinical trials: Is blinding necessary? Control Clin Trials 1996, I7: I- I2

16. Moher D, Jones A, Lepage L, for the CONSORT group: Use of the CONSORT statement and quality of reports of randomized trials: A comparative before-and-after evaluation. JAMA 200I, 285: $1992-5$

17. Linde $K$, Jonas WB, Melchart D, Willich S: The methodological quality of randomized controlled trials of homeopathy, herbal medicines and acupuncture. Int J Epidemiol 200I, 30:S26-3I

18. O'Toole LB: MRC uses checklist similar to consorts. BM] I997, 3 | 4: | | 27

19. loannidis JPA, Lau J: Completeness of safety reporting in randomized trials: an evaluation of $\mathbf{7}$ medical areas. JAMA 200I, 285:437-443

20. loannidis JPA, Contopoulos-loannidis DG: Reporting of safety data from randomised trials. Lancet 1998, 352:1752-1753

21. Liberati A, Himel HN, Chalmers TC: A quality assessment of randomized control trials of primary treatment of breast cancer. J Clin Oncol 1986, 4:942-51

22. Hadhazy V, Ezzo J, Berman B: How valuable is effort to contact authors to obtain missing data in systematic reviews. Presented at the VII Cochrane Colloquium, Rome, Italy. 1999

23. Moher $D$, Fortin $P$, Jadad $A R$, Jüni $P$, Klassen $T$, Le Lorier J, Liberati $A$, Linde K, Penna A: Completeness of reporting of trials published in languages other than English: implications for the conduct of systematic reviews. The Lancet. 1996, 347:363-366

Publish with BioMed Central and every scientist can read your work free of charge

"BioMedcentral will be the most significant development for disseminating the results of biomedical research in our lifetime."

Paul Nurse, Director-General, Imperial Cancer Research Fund

Publish with BMC and your research papers will be:

- available free of charge to the entire biomedical community

- peer reviewed and published immediately upon acceptance

- cited in PubMed and archived on PubMed Central

- yours - you keep the copyright 